\title{
Neural Correlates of Motor Recovery Measured by SPECT at Six Months After Basal Ganglia Stroke
}

\author{
Ji Won Choi, MD' ${ }^{1}$, Myoung Hyoun Kim, MD², Soon-Ah Park, MD², Deok Su Sin, MD' ${ }^{1}$, Min-Su Kim, MD
}

\author{
Departments of ${ }^{1}$ Rehabilitation Medicine and ${ }^{2}$ Nuclear Medicine, \\ Wonkwang University School of Medicine and Hospital, Iksan, Korea
}

\begin{abstract}
Objective To investigate neural correlates associated with recovery of motor function over 6 months in patients with basal ganglia (BG) stroke using acetazolamide (ACZ) stress brain-perfusion single-photon emission computed tomography (SPECT).

Methods Medical records of 22 patients presenting first-ever BG stroke were retrospectively reviewed. Regional cerebral blood flow (CBF) and cerebrovascular reserve (CVR) were measured for 9 regions in each cerebral hemisphere (primary motor cortex, supplementary motor area, premotor cortex, prefrontal cortex, temporal lobe, parietal lobe, occipital lobe, BG, and thalamus). The Fugl-Meyer Assessment (FMA) motor score was used to assess motor function.

Results After ACZ injection, CBF of all regions of interest (ROIs) increased compared with baseline. Baseline CBF of all ROIs was not significantly correlated with changes in FMA upper or lower motor score. However, multivariate analysis revealed CVR was significantly associated with change in FMA upper score in the ipsilateral primary motor cortex $\left(\mathrm{R}^{2}=0.216, \mathrm{p}=0.017\right)$, the ipsilateral parietal lobe $\left(\mathrm{R}^{2}=0.135, \mathrm{p}=0.029\right)$, and the contralateral primary motor cortex $\left(\mathrm{R}^{2}=0.210, \mathrm{p}=0.041\right)$.

Conclusion CVR in the bilateral primary motor cortex and ipsilateral parietal lobe was associated with restoration of upper motor function 6 months after BG stroke. SPECT is a readily available imaging modality useful in studying brain residual function in patients with BG stroke.
\end{abstract}

Keywords Acetazolamide, Basal ganglia, Recovery of function, Stroke, Single photon emission computed tomography

\section{INTRODUCTION}

Rehabilitation therapy contributes to neural reorgani- zation of regions involved in motor recovery following stroke. For this reason, ability to live independently after stroke depends largely on improvement of motor func-

Received March 29, 2017; Accepted May 22, 2017

Corresponding author: Min-Su Kim

Department of Rehabilitation Medicine, Wonkwang University School of Medicine \& Hospital, 895 Muwang-ro, Iksan 54538, Korea. Tel: +82-63-8591610, Fax: +82-63-843-1385, E-mail: helmaine@naver.com

ORCID: Ji Won Choi (http://orcid.org/0000-0002-5339-4854); Myoung Hyoun Kim (http://orcid.org/0000-0002-4541-8019); Soon-Ah Park (http:// orcid.org/0000-0002-8269-8865); Deok Su Sin (http://orcid.org/0000-0001-5920-6925); Min-Su Kim (http://orcid.org/0000-0001-9954-1445).

(c) This is an open-access article distributed under the terms of the Creative Commons Attribution Non-Commercial License (http://creativecommons.org/ licenses/by-nc/4.0) which permits unrestricted noncommercial use, distribution, and reproduction in any medium, provided the original work is properly cited. Copyright (C) 2017 by Korean Academy of Rehabilitation Medicine 
tion through rehabilitation therapy [1]. Basal ganglia (BG) are involved in higher-order motor control such as movement planning and execution of complex motor synergies [2]. In addition, BG play a critical role in motor skill acquisition by coordinating dynamic interaction among multiple neural networks in frontal and parietal cortices [3]. Thus, information on brain plasticity during motor recovery after BG lesion stroke may help clarify motor function rehabilitation. This study therefore included only BG stroke patients in to limit pathological heterogeneity that may occur in stroke patients with cerebral cortex involvement.

Development of functional imaging techniques has greatly advanced our understanding of neural mechanisms involved in rehabilitation. In particular, diffusion tensor imaging (DTI) and functional magnetic resonance imaging (fMRI) are frequently used to investigate changes in neural activity [4]. In studies using these imaging modalities, patients with good or complete motor recovery display activation patterns like healthy control subjects $[5,6]$. More recent data has emphasized the significance of corticospinal tract integrity for successful motor recovery $[7,8]$.

Currently, single-photon emission computed tomography (SPECT) is the most readily available nuclear medicine technique for assessment of cerebral hemodynamics [9]. Despite having lower spatial resolution, SPECT has higher sensitivity than perfusion-weighted imaging for detecting hypoperfused tissue [10]. Furthermore, SPECT is relatively inexpensive compared to fMRI and DTI and does not require a specialist for data analysis.

Several studies have used SPECT to investigate motor recovery in patients with stroke. Kononen et al. [11] used SPECT to assess stroke patients at rest before and 2 weeks after constraint therapy. Results of this study revealed increased perfusion in the precentral gyrus, premotor cortex, frontal cortex, and superior frontal gyrus of the affected hemisphere. Takekawa et al. [12] reported that improvement in perfusion of the superior and middle frontal areas was related to recovery of upper limb motor function in post-stroke patients following repetitive transcranial magnetic stimulation (rTMS). However, both studies evaluated only upper extremity function and effect of intervention such as rTMS and constraint-induced movement therapy. Park et al. [13] suggested that recovery of gross motor function was correlated with baseline regional cerebral blood flow (CBF) and cerebrovascular reserve (CVR) in the affected supratentorial hemisphere of stroke patients. This study evaluated CVR only in patients with ischemic stroke, suggesting that CVR of the affected cerebral hemisphere is a significant predictor of recovery, regardless of lesion location. Despite widespread use of SPECT, there have been few SPECT studies of patients with BG stroke that attempted to identify areas associated with long-term functional recovery.

Thus, the purpose of this study was to use SPECT to identify regions that are associated with motor recovery 6 months after BG stroke, and to analyze the relationship between CVR and change in motor function in patients with BG stroke.

\section{MATERIALS AND METHODS}

\section{Subjects}

We used a retrospective study design to analyze medical records of patients presented with BG stroke to Wonkwang University Hospital from January 2013 to December 2015. All patients underwent brain computed tomography (CT) or diffusion brain MRI within 24 hours of initial onset and were examined by a neurosurgeon or neurologist. Of these patients, only those who were first-ever stroke, were referred to the rehabilitation department and underwent brain SPECT during the hospitalization period were included in this study.

Exclusion criteria included the following: (1) concurrent intracerebral hemorrhage or cerebral infarct in other regions; (2) past medical history of diseases impacting overt motor outcomes, such as Parkinson disease, spinal cord injury, and motor neuron disease; (3) any clinically significant or unstable medical condition or any neuropsychiatric comorbidity other than stroke. This study was approved by the Institutional Review Board of Wonkwang University Hospital (No. WKUH 201607-HRE-080).

\section{Brain perfusion SPECT study}

Baseline SPECT images and acetazolamide (ACZ) stress brain-perfusion SPECT images were obtained from all patients after admission. ACZ stress brain-perfusion SPECT (ACZ-SPECT) consists of a baseline SPECT image and an image acquired after ACZ administration. Both image types can be obtained during the course of 1 day [14]. 
Baseline SPECT was obtained 60 minutes after intravenous injection of $555 \mathrm{MBq}$ of $99 \mathrm{mTc}$-hexamethylpropyleneamineoxime (HMPAO) using a dual-head detector gamma camera (Millennium MG; GE Medical System, Milwaukee, WI, USA). Fifteen minutes before the end of the baseline SPECT, $15 \mathrm{mg} / \mathrm{kg}$ of ACZ was injected intravenously, and another 1,665 MBq of 99mTc-HMPAO was injected following completion of the baseline SPECT. ACZ-SPECT was conducted 60 minutes after the second administration of $99 \mathrm{mTc}$-HMPAO.

SPECT images were acquired using low-energy highresolution parallel-hole collimator with a $\gamma$-ray energy window of $140 \mathrm{keV} \pm 10 \%$ in a $128 \times 128$ matrix size. Sixtyfour step-and-shoot images were obtained with intervals of $2.8^{\circ}$ for 15 seconds per step. SPECT images were reconstructed with two iterations using ordered subset expectation maximization algorithm and regularized with a Butterworth filter.

\section{Quantitative assessment of regional CBF and CVR}

$\mathrm{CBF}$ was determined in each of 9 regions (primary mo- tor cortex, supplementary motor area [SMA], premotor cortex, prefrontal cortex, temporal lobe, parietal lobe, occipital lobe, BG, and thalamus). The reasons for selecting these each 9 areas are as follow. First, we tried to assess effects of brain area on motor recovery in as many cortical areas as possible, including the motor cortex and other areas associated with brain circuits for spontaneous movement [15]. Second, diaschisis has a significant effect on stroke recovery [16]. To minimize this effect, the infratentorial lesion, cerebellum, was excluded.

Regions of interest (ROIs) on axial, coronal and sagittal images were identified, and counts per pixel were measured and averaged for each ROI. Finally, mean counts per pixel were normalized using total counts of the brain to minimize confounding factors such as differences in injected dose, time interval of imaging, and individual bio-distribution (Fig. 1).

ACZ is a carbonic anhydrase inhibitor that penetrates the blood-brain barrier slowly and acts as a cerebral vasodilator agent [9]. Inhibition of carbonic anhydrase causes carbonic acidosis, that considerably increases re-
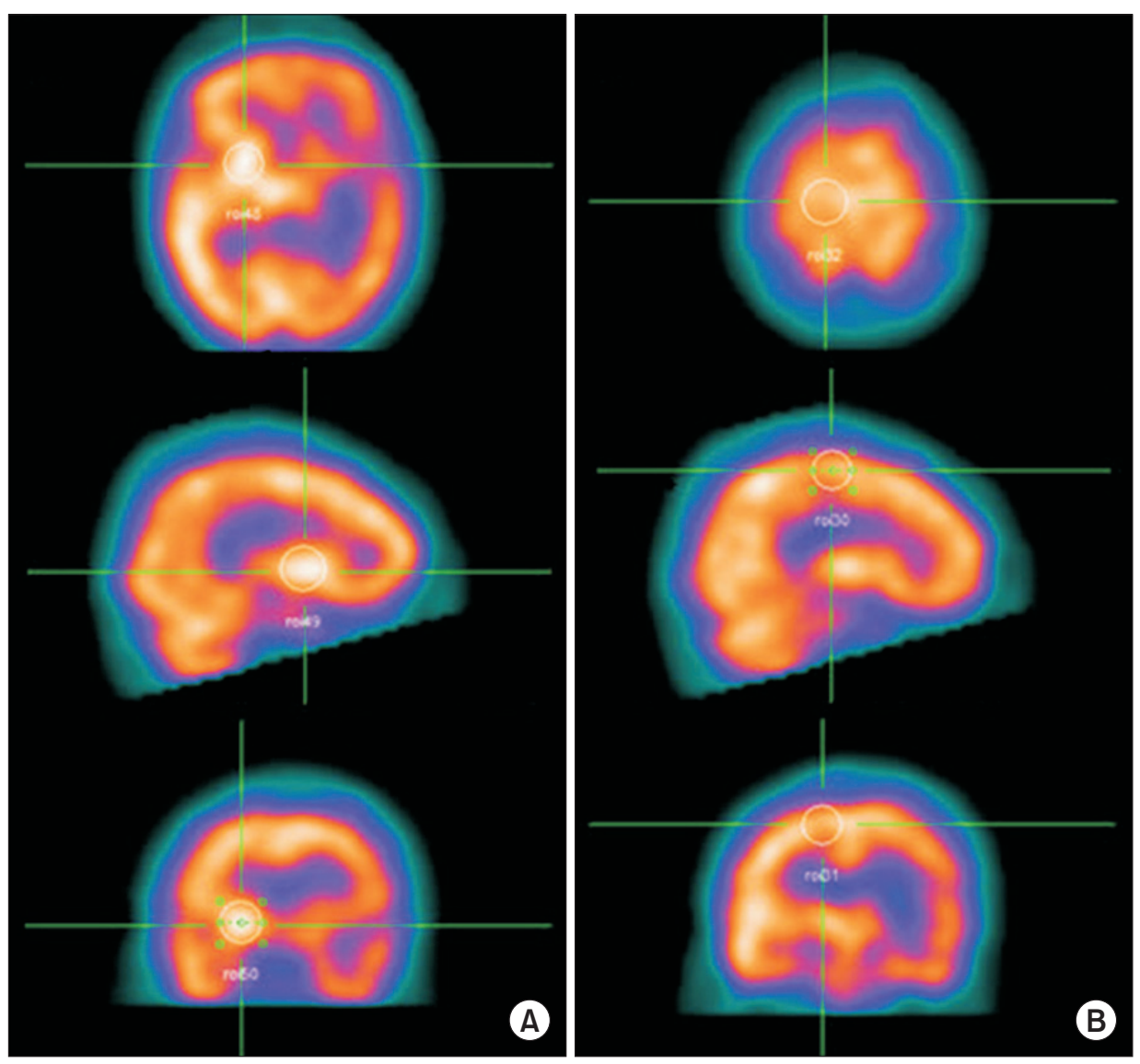

Fig. 1. Measurement of the baseline cerebral blood flow in each region of interest (ROI) using SPECT. ROIs on the axial, coronal, and sagittal images were recognized, and then the counts per pixel of ROIs were quantitatively measured and averaged. Finally, the mean counts per pixel were normalized versus the total counts for the brain. (A) Basal ganglia. (B) Primary motor cortex. 
gional CBF $[17,18]$; up to $30 \%-60 \%$ is achieved in healthy subjects [19]. A significant increase in local CBF after administration of ACZ signifies collateral circulation is well developed even with significant stenosis of the vessel; thus, normal cerebral perfusion pressure and vascular reserve are maintained. Therefore, perfusion SPECT provides valuable information in acute stroke regarding treatment choice and prognosis [20]. CVR was defined as preserved if CBF of stress SPECT was greater than that of baseline SPECT (Fig. 2).

$$
\text { CVR }(\%)=\frac{\Delta \text { Regional CBF }(\text { stress flow }- \text { baseline flow })}{\text { Regional } C B F(\text { baseline flow })} \times 100
$$

\section{Assessment of motor function}

Several clinical and functional parameters that may impact motor outcome 6 months after BG stroke were investigated. We retrospectively reviewed clinical assessments and collected information on demographics, type of stroke, affected hemisphere, and time between stroke onset and brain SPECT.

The Fugl-Meyer Assessment (FMA) applied to each patient at the time of SPECT imaging and 6 months after stroke was used to assess motor function. The FMA consists of 5 domains (motor, sensory, balance, range of motion, and joint pain); however, for this study we only used the motor domain score to evaluate comprehensive motor function. The FMA motor domain ranges in score from 0-100 and includes an assessment of upper and lower extremities. Upper limb motor score ranges from 0-66, while the lower limb motor score ranges from 0-34.

Activities of daily living were assessed by the Korean version of the Modified Barthel Index. Additionally, the
Korean version of the Mini-Mental Status Examination conducted at the time of SPECT was used as a measure of cognitive function.

\section{Statistical analysis}

Baseline characteristics of the study group were summarized as numbers and percentages for categorical variables. Continuous variables were summarized as mean and standard deviation. Distribution of data was assessed with the Kolmogorov-Smirnov test prior to group comparisons for quantitative variables. Simple correlation was used to identify confounding factors that may impact motor function recovery. Univariate regression analysis was used to analyze the relationship between baseline CBF and changes in FMA upper and lower motor score. Multivariate regression analysis was used to identify the relationship between regional CVR and changes in FMA upper and lower motor score. Analysis was conducted using SPSS ver. 22.0 (IBM, Armonk, NY, USA). The pvalues $<0.05$ were considered statistically significant.

\section{RESULTS}

A total of 22 patients ( $60.3 \pm 12.4$ years) were included in this study. Mean time from stroke onset to SPECT imaging was $28.7 \pm 11.6$ days. Nine of 22 patients had a cerebral infarction, while 13 patients had a cerebral hemorrhage. Average FMA upper motor score was $22.6 \pm 13.6$ and average lower motor score was $18.2 \pm 7.2$ (Table 1 ).

Following ACZ injection, the CBF of all the ROIs was increased over baseline. A mean CVR increase of $12.3 \%$ was observed across all regions (Table 2).
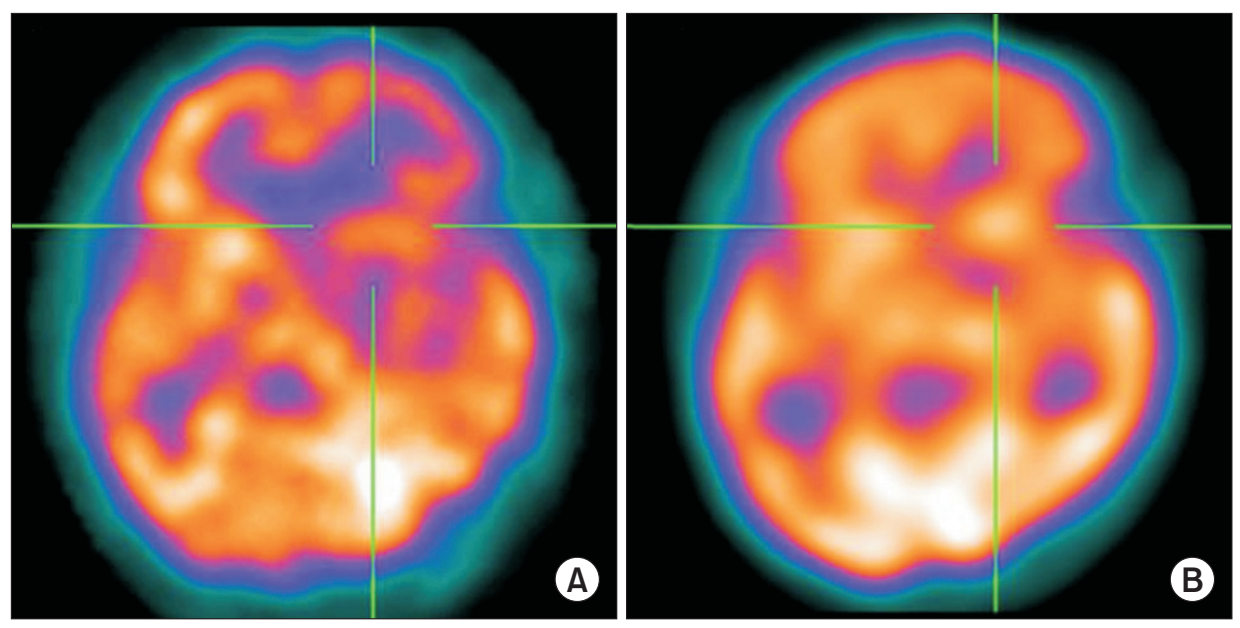

Fig. 2. Calculation of the cerebrovascular reserve (CVR). After acetazolamide injection, CVR was measured as a percentage of the recovery of the blood flow relative to the baseline. (A) There are areas of severe hypoperfusion in the left basal ganglia at baseline. (B) After injection, a significant improvement of perfusion was demonstrated, suggesting preserved CVR. 
We conducted simple correlation analyses to identify confounds in the relationship between $\mathrm{CBF}$ and motor recovery and found that age $\left(\mathrm{R}^{2}=0.102, \mathrm{p}=0.035\right)$, FMA upper motor score $\left(\mathrm{R}^{2}=0.345, \mathrm{p}=0.025\right)$ and FMA lower

Table 1. Baseline characteristics of the subjects $(n=22)$

\begin{tabular}{|cc}
\hline \multicolumn{1}{|c}{ characteristic } & Value \\
\hline Age $(\mathrm{yr})$ & $60.3 \pm 12.4$ \\
\hline Sex & $16(72.7)$ \\
\hline Male & $6(27.3)$ \\
\hline Female & \\
\hline Stroke type & $9(40.9)$ \\
\hline Infarction & $13(59.1)$ \\
\hline Hemorrhage & $11(50.0)$ \\
\hline Lesion side & $11(50.0)$ \\
\hline Left & $28.7 \pm 11.6$ \\
\hline Right & \\
\hline Elapsed time for SPECT (day) & $22.6 \pm 13.6$ \\
\hline Motor score of FMA & $18.18 \pm 7.2$ \\
\hline Upper, affected & $49 \pm 16.3$ \\
\hline Lower, affected & $22.9 \pm 8.3$ \\
\hline K-MBI & \\
\hline K-MMSE &
\end{tabular}

Values are presented as mean \pm standard deviation or number (\%).

SPECT, single-photon emission computed tomography; FMA, Fugl-Meyer Assessment; K-MBI, Korean version of Modified Barthel Index; K-MMSE, Korean version of Mini-Mental Status Examination. motor score $\left(\mathrm{R}^{2}=0.215, \mathrm{p}=0.039\right)$ significantly correlated (Table 3 ). After adjusting for these confounding factors, we analyzed the relationship between changes of FMA upper and lower motor score and baseline CBF using univariate regression analysis. Baseline CBF was not correlated with changes in FMA motor upper and lower score in any ROI (Table 4). After ACZ administration,

Table 3. Relationship between motor outcome 6 months post-stroke and probable confounding factors for motor recovery

\begin{tabular}{lcc}
\hline \multicolumn{1}{c}{ Variable } & \multicolumn{2}{c}{$\Delta$ FMA } \\
\cline { 2 - 3 } & $\begin{array}{c}\text { Coefficient of } \\
\text { correlation }\end{array}$ & p-value \\
\hline Age & 0.102 & $0.035^{*}$ \\
Sex & 0.012 & 0.957 \\
Stroke type & 0.327 & 0.138 \\
Lesion side & 0.324 & 0.142 \\
Elapsed time for SPECT (day) & 0.218 & 0.329 \\
Motor score of FMA & & \\
$\quad$ Upper, affected & 0.345 & $0.025^{*}$ \\
\hline Lower, affected & 0.215 & $0.039^{*}$ \\
K-MBI & 0.013 & 0.859 \\
K-MMSE & 0.011 & 0.723 \\
\hline
\end{tabular}

SPECT, single-photon emission computed tomography; FMA, Fugl-Meyer Assessment; K-MBI, Korean version of Modified Barthel Index; K-MMSE, Korean version of Mini-Mental Status Examination.

${ }^{*} \mathrm{p}<0.05$.

Table 2. Changes of the baseline cerebral blood flow after acetazolamide administration according to regions of interest

\begin{tabular}{|c|c|c|c|c|c|c|}
\hline \multirow[b]{2}{*}{ ROI } & \multicolumn{3}{|c|}{ Ipsilateral } & \multicolumn{3}{|c|}{ Contralateral } \\
\hline & $\begin{array}{c}\text { Base CBF } \\
\left(\times 10^{6}\right)\end{array}$ & $\begin{array}{l}\text { Stress CBF } \\
\quad\left(\times 10^{6}\right)\end{array}$ & CVR (\%) & $\begin{array}{c}\text { Base CBF } \\
\left(\times 10^{6}\right)\end{array}$ & $\begin{array}{l}\text { Stress CBF } \\
\left(\times 10^{6}\right)\end{array}$ & CVR (\%) \\
\hline BG & 43.0 & 46.0 & 14.2 & 49.0 & 52.3 & 11.7 \\
\hline Thalamus & 41.0 & 44.0 & 12.8 & 45.0 & 47.6 & 11.2 \\
\hline Prefrontal cortex & 40.0 & 44.0 & 12.6 & 44.0 & 47.2 & 11.7 \\
\hline SMA & 41.0 & 45.0 & 12.1 & 43.0 & 46.9 & 12.4 \\
\hline Premotor cortex & 40.0 & 47.0 & 20.8 & 44.0 & 47.0 & 9.7 \\
\hline M1 & 39.0 & 43.0 & 13.7 & 42.0 & 45.5 & 11.0 \\
\hline Parietal lobe & 40.0 & 44.0 & 14.1 & 44.0 & 47.6 & 12.3 \\
\hline Temporal lobe & 38.0 & 42.0 & 13.3 & 42.0 & 45.2 & 10.1 \\
\hline Occipital lobe & 46.0 & 49.0 & 9.9 & 48.0 & 49.9 & 7.2 \\
\hline
\end{tabular}

ROI, region of interest; CBF, cerebral blood flow; CVR, cerebrovascular reserve; BG, basal ganglia; SMA, supplementary motor area; M1, primary motor cortex. 
Table 4. Correlation between the baseline cerebral blood flow of each ROI and the changes of FMA motor score

\begin{tabular}{|c|c|c|c|c|}
\hline & \multicolumn{2}{|c|}{$\Delta$ FMA (upper) } & \multicolumn{2}{|c|}{$\Delta$ FMA (lower) } \\
\hline & Coefficient of correlation & p-value & Coefficient of correlation & p-value \\
\hline \multicolumn{5}{|l|}{ Ipsilateral } \\
\hline BG & 0.199 & 0.065 & 0.002 & 0.849 \\
\hline Thalamus & 0.025 & 0.521 & 0.051 & 0.352 \\
\hline Prefrontal cortex & 0.105 & 0.176 & 0.026 & 0.512 \\
\hline SMA & 0.154 & 0.097 & 0.001 & 0.923 \\
\hline Premotor cortex & 0.116 & 0.153 & 0.001 & 0.879 \\
\hline M1 & 0.002 & 0.853 & 0.024 & 0.523 \\
\hline Parietal lobe & 0.001 & 0.918 & 0.104 & 0.177 \\
\hline Temporal lobe & 0.034 & 0.447 & 0.002 & 0.988 \\
\hline Occipital lobe & 0.013 & 0.649 & 0.111 & 0.164 \\
\hline \multicolumn{5}{|l|}{ Contralateral } \\
\hline BG & 0.020 & 0.568 & 0.002 & 0.953 \\
\hline Thalamus & 0.096 & 0.196 & 0.031 & 0.965 \\
\hline Prefrontal cortex & 0.118 & 0.151 & 0.044 & 0.389 \\
\hline SMA & 0.003 & 0.840 & 0.010 & 0.412 \\
\hline Premotor cortex & 0.104 & 0.177 & 0.068 & 0.282 \\
\hline M1 & 0.021 & 0.556 & 0.104 & 0.176 \\
\hline Parietal lobe & 0.086 & 0.222 & 0.024 & 0.526 \\
\hline Temporal lobe & 0.087 & 0.221 & 0.060 & 0.314 \\
\hline Occipital lobe & 0.009 & 0.694 & 0.154 & 0.096 \\
\hline
\end{tabular}

ROI, region of interest; $\triangle \mathrm{FMA}$, difference of Fugl-Meyer Assessment between initial and 6 months post-stroke value; BG, basal ganglia; SMA, supplementary motor area; M1, primary motor cortex.

change in FMA lower motor was not correlated with ROI (Table 5). However, CVR of the ipsilateral primary motor area $\left(M 1, R^{2}=0.216, p=0.017\right)$, the parietal lobe $\left(R^{2}=0.135\right.$, $\mathrm{p}=0.029)$ and contralateral $\mathrm{M} 1\left(\mathrm{R}^{2}=0.210, \mathrm{p}=0.041\right)$ were all significantly correlated with the change of FMA upper motor score (Table 5, Fig. 3).

\section{DISCUSSION}

In this study, we investigated the relationship between motor recovery at 6 months post-BG stroke and CVR. Results reveal that CVR of the bilateral M1 and the ipsilateral parietal lobe shortly after BG stroke predict upper motor recovery at 6 months post-stroke. However, in this study, lower limb FMA score was not significantly associated with motor recovery. We suggest that the reason for this difference is as follows.

First, there is a fundamental difference between nervous control of upper and lower limb movement. Upper limb movement is under cerebral-cerebellar control [21], while gait of lower limbs are under control of spinal interneurons in the central pattern generator [22]. Second, there is a difference in behavior pattern between upper and lower limbs. Arms and hands are used for voluntary reaching, grasping, and manipulation of objects, whereas legs are mostly involved in locomotion [23]. Third, in the motor score of FMA, upper extremity score is 66 points, while lower extremity score is $\mathbf{3 4}$ points. Lower extremity motor score is lower than upper extremity, so ability to assess functional recovery may be less sensitive in lower limbs compared to upper limbs.

Many studies have demonstrated that several brain regions and their interaction contribute to recovery of motor function after stroke. Specifically, several fMRI studies have revealed that ipsilateral M1 is crucial for motor recovery and functioning after stroke [24-26]. Meta-analysis of data from patients with chronic stroke revealed that changes in patterns of ipsilateral medialpremotor and M1 activity predict motor recovery, suggesting that these areas may be excellent biomarkers for 
Table 5. Relationship between the cerebrovascular reserve and the change of motor outcomes after 6 months

\begin{tabular}{|c|c|c|c|c|}
\hline & \multicolumn{2}{|c|}{$\Delta$ FMA (upper) } & \multicolumn{2}{|c|}{$\Delta$ FMA (lower) } \\
\hline & Coefficient of correlation & p-value & Coefficient of correlation & p-value \\
\hline \multicolumn{5}{|l|}{ Ipsilateral } \\
\hline BG & 0.199 & 0.065 & 0.002 & 0.849 \\
\hline Thalamus & 0.025 & 0.521 & 0.051 & 0.352 \\
\hline Prefrontal cortex & 0.105 & 0.176 & 0.026 & 0.512 \\
\hline SMA & 0.154 & 0.097 & 0.015 & 0.923 \\
\hline Premotor cortex & 0.116 & 0.153 & 0.021 & 0.879 \\
\hline M1 & 0.216 & $0.017^{*}$ & 0.024 & 0.523 \\
\hline Parietal lobe & 0.135 & $0.029^{*}$ & 0.104 & 0.177 \\
\hline Temporal lobe & 0.034 & 0.447 & 0.031 & 0.988 \\
\hline Occipital lobe & 0.013 & 0.649 & 0.111 & 0.164 \\
\hline \multicolumn{5}{|l|}{ Contralateral } \\
\hline BG & 0.020 & 0.568 & 0.013 & 0.953 \\
\hline Thalamus & 0.096 & 0.196 & 0.141 & 0.965 \\
\hline Prefrontal cortex & 0.118 & 0.151 & 0.044 & 0.389 \\
\hline SMA & 0.003 & 0.840 & 0.040 & 0.412 \\
\hline Premotor cortex & 0.104 & 0.177 & 0.068 & 0.282 \\
\hline M1 & 0.210 & $0.041^{*}$ & 0.104 & 0.179 \\
\hline Parietal lobe & 0.086 & 0.222 & 0.024 & 0.526 \\
\hline Temporal lobe & 0.087 & 0.221 & 0.060 & 0.314 \\
\hline Occipital lobe & 0.009 & 0.694 & 0.154 & 0.196 \\
\hline
\end{tabular}

$\triangle$ FMA, difference of Fugl-Meyer Assessment between initial and 6 months post-stroke value; BG, basal ganglia; SMA, supplementary motor area; Ml, primary motor cortex.

${ }^{*} \mathrm{p}<0.05$.

sensorimotor recovery [25]. Another study reported that greater functional connectivity of ipsilateral M1 with contralateral thalamus, SMA, and middle frontal gyrus at stroke onset predicts motor recovery at 6 months poststroke [26]. Consistent with these studies, we report that CVR of ipsilateral M1 was significantly associated with motor recovery in our sample of BG stroke patients.

In addition to these findings, we also report that CVR in contralateral M1 predicted motor recovery post-stroke. Longitudinal fMRI studies have revealed that gradually increasing activity in the contralateral $\mathrm{Ml}$ and premotor cortex is positively correlated with functional recovery in severely impacted patients. This indicates that stroke promotes cortical reorganization to support motor function of the affected hand [27]. Additionally, fMRI data suggest that rTMS of contralateral M1 may normalize neural activation within the cortical motor network following subcortical stroke [28]. Findings of these studies and our study demonstrate the significance of contralateral M1 activity in motor function recovery post-stroke.

The parietal lobe has recently gained attention for its role in recovery of motor function after stroke. Patients with stroke reveal enhanced reciprocal connectivity between M1 and anterior intraparietal sulcus of the ipsilateral hemisphere compared with healthy controls [29]. It has also been reported that integrity of tracts that connect the ventral premotor cortex, M1, anterior intraparietal sulcus, and ventral premotor cortex is correlated with motor function [30]. Here, we demonstrate the parietal lobe plays a crucial role in motor recovery in patients with BG stroke.

In addition to bilateral $\mathrm{Ml}$ and ipsilateral parietal lobe findings discussed above, other studies have demonstrated that premotor areas and the SMA are associated with motor recovery after stroke. Following a stroke, the brain reorganizes by engaging a dynamic neural network that includes the premotor area of both hemispheres [31,32]. In an investigation of motor cortical areas that participate 

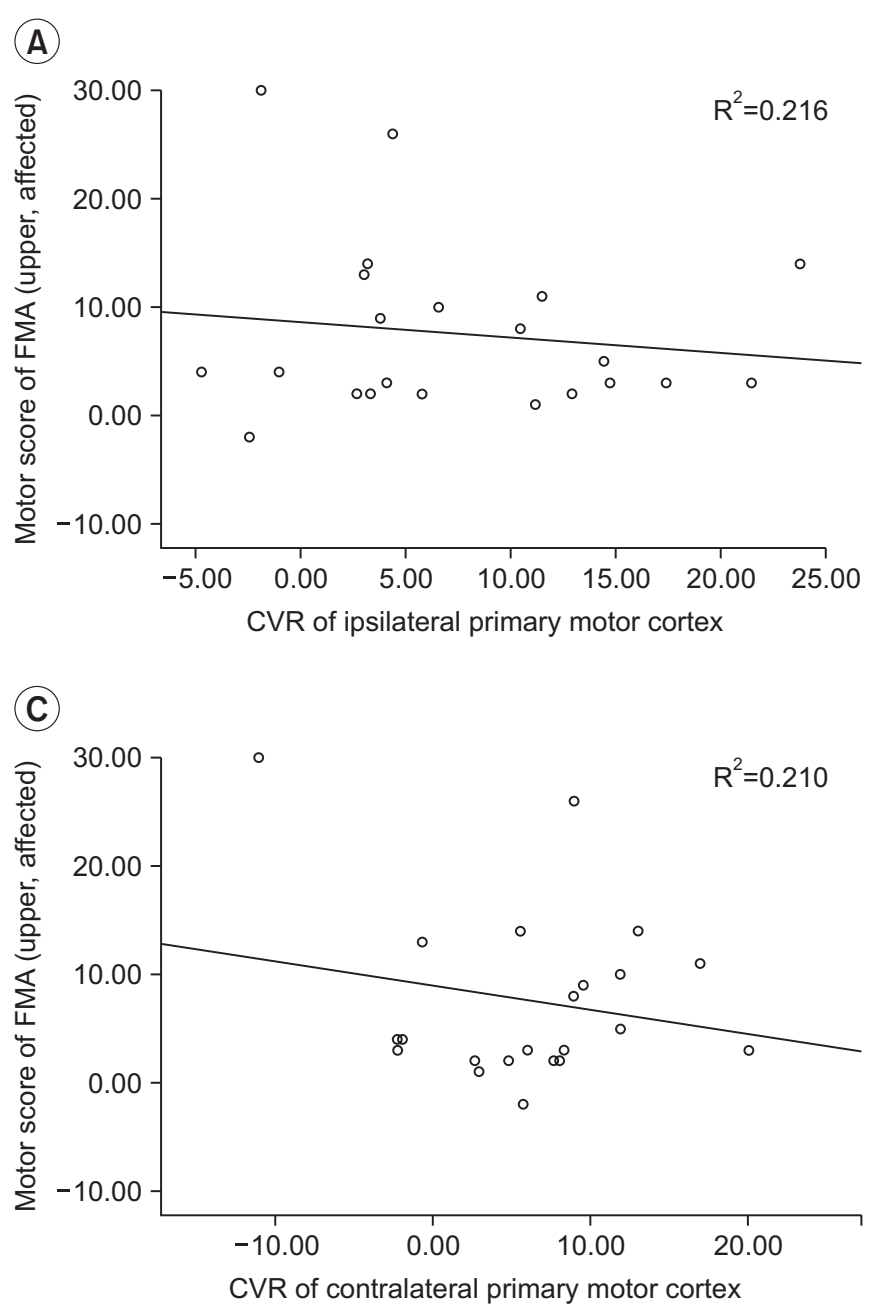

in motor imagery performance in stroke patients, it was found that bilateral SMA and ipsilateral M1 are useful for monitoring compliance in stroke patients [33]. In this study, we did not detect correlation between either the SMA or premotor area and recovery of motor function.

SPECT and fMRI are functional neuroimaging modality for assessing brain activity [34], and the same results as in other fMRI studies for stroke patients were noted in this study. However, since SPECT measures CBF whereas fMRI evaluates of brain metabolism, it is a limitation of this study to apply the above fMRI study results directly to this study. However, our results suggest that the Ml and ipsilateral parietal lobe are of pre-eminent significance. More research is needed to clarify the cause of discrepancy between our results and those reported by others to identify which brain regions and their interaction are most critical for motor recovery after stroke.

SPECT is the most readily available nuclear medicine

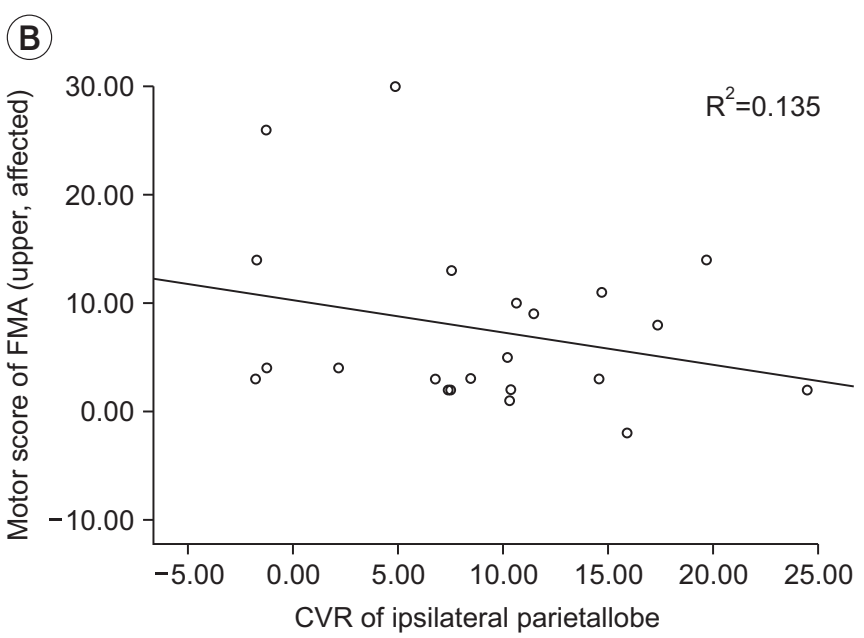

Fig. 3. Neural correlates of motor recovery 6 months post-BG stroke. The restoration of blood flow in bilateral primary motor cortex and ipsilateral parietal lobe were significantly related to the improvement of upper motor function. (A) Ipsilateral primary motor cortex. (B) Ipsilateral parietal lobe. (C) Contralateral primary motor cortex. BG, basal ganglia; CVR, cerebrovascular reserve; FMA, Fugl-Meyer Assessment.

technique for assessment of cerebral hemodynamics [9], and is less expensive than either fMRI or DTI. SPECT can detect changes in blood flow with high sensitivity (61\%$74 \%$ ) and specificity (88\%-98\%) immediately after onset of symptoms [35]. Several studies have demonstrated usefulness of brain SPECT in conjunction with ACZ for evaluation of vascular reactivity in patients with various types of vaso-occlusive disease [9]. Based on those advantages, we selected SPECT for this study.

This study has several limitations. First, because this was a retrospective case-control study in a center, it is possible that there was selection bias. This study also had a limited number of participants. Secondly, because SPECT has less resolution than fMRI, anatomical localization was difficult in the lobe, making distinction between primary somatosensory cortex and posterior parietal cortex challenging. Thus, in the future, a larger, multi-center prospective study that uses higher resolu- 
tion SPECT techniques than are available today will be necessary to understand motor networks that mediate long-term recovery in patients with BG stroke.

In conclusion, CVR in bilateral M1 and ipsilateral parietal lobe predicted restoration of upper motor function after 6 months in BG stroke patients. This study demonstrates the usefulness of SPECT imaging in determining residual brain function in $\mathrm{BG}$ stroke patients.

\section{CONFLICT OF INTEREST}

No potential conflict of interest relevant to this article was reported.

\section{REFERENCES}

1. Stinear C. Prediction of recovery of motor function after stroke. Lancet Neurol 2010;9:1228-32.

2. Groenewegen HJ. The basal ganglia and motor control. Neural Plast 2003;10:107-20.

3. Hikosaka O, Nakamura K, Sakai K, Nakahara H. Central mechanisms of motor skill learning. Curr Opin Neurobiol 2002;12:217-22.

4. Grefkes C, Fink GR. Connectivity-based approaches in stroke and recovery of function. Lancet Neurol 2014;13:206-16.

5. Carey LM, Abbott DF, Egan GF, O'Keefe GJ, Jackson GD, Bernhardt J, et al. Evolution of brain activation with good and poor motor recovery after stroke. Neurorehabil Neural Repair 2006;20:24-41.

6. Calautti C, Leroy F, Guincestre JY, Baron JC. Dynamics of motor network overactivation after striatocapsular stroke: a longitudinal PET study using a fixed-performance paradigm. Stroke 2001;32:2534-42.

7. Ward NS, Newton JM, Swayne OB, Lee L, Thompson AJ, Greenwood RJ, et al. Motor system activation after subcortical stroke depends on corticospinal system integrity. Brain 2006;129(Pt 3):809-19.

8. Lindenberg R, Renga V, Zhu LL, Betzler F, Alsop D, Schlaug G. Structural integrity of corticospinal motor fibers predicts motor impairment in chronic stroke. Neurology 2010;74:280-7.

9. Vagal AS, Leach JL, Fernandez-Ulloa M, Zuccarello M. The acetazolamide challenge: techniques and applications in the evaluation of chronic cerebral ischemia. AJNR Am J Neuroradiol 2009;30:876-84.
10. Nuutinen J, Liu Y, Laakso MP, Karonen JO, Vanninen EJ, Kuikka JT, et al. Perfusion differences on SPECT and PWI in patients with acute ischemic stroke. Neuroradiology 2009;51:687-95.

11. Kononen M, Kuikka JT, Husso-Saastamoinen M, Vanninen E, Vanninen R, Soimakallio S, et al. Increased perfusion in motor areas after constraint-induced movement therapy in chronic stroke: a single-photon emission computerized tomography study. J Cereb Blood Flow Metab 2005;25:1668-74.

12. Takekawa T, Kakuda W, Uchiyama M, Ikegaya M, Abo M. Brain perfusion and upper limb motor function: a pilot study on the correlation between evolution of asymmetry in cerebral blood flow and improvement in Fugl-Meyer Assessment score after rTMS in chronic post-stroke patients. J Neuroradiol 2014;41:177-83.

13. Park SA, Park HI, Kim D, Yang CY, Zhang LQ. The prediction of gross motor outcome using cerebrovascular reserve measured by acetazolamide-challenged SPECT. NeuroRehabilitation 2012;30:359-67.

14. Hattori N, Yonekura Y, Tanaka F, Fujita T, Wang J, Ishizu K, et al. One-day protocol for cerebral perfusion reserve with acetazolamide. J Nucl Med 1996;37:205761.

15. Haggard P. Human volition: towards a neuroscience of will. Nat Rev Neurosci 2008;9:934-46.

16. Seitz RJ, Azari NP, Knorr U, Binkofski F, Herzog H, Freund HJ. The role of diaschisis in stroke recovery. Stroke 1999;30:1844-50.

17. Andaluz N, Choutka O, Vagal A, Strunk R, Zuccarello M. Patient selection for revascularization procedures in adult Moyamoya disease based on dynamic perfusion computerized tomography with acetazolamide challenge (PCTA). Neurosurg Rev 2010;33:225-32.

18. Iida H, Nakagawara J, Hayashida K, Fukushima K, Watabe $\mathrm{H}$, Koshino K, et al. Multicenter evaluation of a standardized protocol for rest and acetazolamide cerebral blood flow assessment using a quantitative SPECT reconstruction program and split-dose 123Iiodoamphetamine. J Nucl Med 2010;51:1624-31.

19. Yonas H, Darby JM, Marks EC, Durham SR, Maxwell C. CBF measured by Xe-CT: approach to analysis and normal values. J Cereb Blood Flow Metab 1991;11:71625.

20. Kapucu OL, Nobili F, Varrone A, Booij J, Vander Borght $\mathrm{T}$, Nagren $\mathrm{K}$, et al. EANM procedure guideline 
for brain perfusion SPECT using 99mTc-labelled radiopharmaceuticals, version 2. Eur J Nucl Med Mol Imaging 2009;36:2093-102.

21. Cramer SC, Parrish TB, Levy RM, Stebbins GT, Ruland SD, Lowry DW, et al. Predicting functional gains in a stroke trial. Stroke 2007;38:2108-14.

22. Hultborn H, Nielsen JB. Spinal control of locomotion: from cat to man. Acta Physiol (Oxf) 2007;189:111-21.

23. Luft AR, Smith GV, Forrester L, Whitall J, Macko RF, Hauser TK, et al. Comparing brain activation associated with isolated upper and lower limb movement across corresponding joints. Hum Brain Mapp 2002;17:131-40.

24. Rehme AK, Eickhoff SB, Wang LE, Fink GR, Grefkes C. Dynamic causal modeling of cortical activity from the acute to the chronic stage after stroke. Neuroimage 2011;55:1147-58.

25. Favre I, Zeffiro TA, Detante O, Krainik A, Hommel M, Jaillard A. Upper limb recovery after stroke is associated with ipsilesional primary motor cortical activity: a meta-analysis. Stroke 2014;45:1077-83.

26. Park CH, Chang WH, Ohn SH, Kim ST, Bang OY, Pascual-Leone A, et al. Longitudinal changes of restingstate functional connectivity during motor recovery after stroke. Stroke 2011;42:1357-62.

27. Rehme AK, Fink GR, von Cramon DY, Grefkes C. The role of the contralesional motor cortex for motor recovery in the early days after stroke assessed with longitudinal FMRI. Cereb Cortex 2011;21:756-68.

28. Nowak DA, Grefkes C, Dafotakis M, Eickhoff S, Kust J, Karbe H, et al. Effects of low-frequency repetitive transcranial magnetic stimulation of the contralesional primary motor cortex on movement kinematics and neural activity in subcortical stroke. Arch Neurol 2008;65:741-7.

29. Schulz R, Buchholz A, Frey BM, Bonstrup M, Cheng B, Thomalla G, et al. Enhanced effective connectivity between primary motor cortex and intraparietal sulcus in well-recovered stroke patients. Stroke 2016;47:4829.

30. Schulz R, Koch P, Zimerman M, Wessel M, Bonstrup M, Thomalla G, et al. Parietofrontal motor pathways and their association with motor function after stroke. Brain 2015;138(Pt 7):1949-60.

31. O'Shea J, Sebastian C, Boorman ED, Johansen-Berg $H$, Rushworth MF. Functional specificity of human premotor-motor cortical interactions during action selection. Eur J Neurosci 2007;26:2085-95.

32. O'Shea J, Johansen-Berg H, Trief D, Gobel S, Rushworth MF. Functionally specific reorganization in human premotor cortex. Neuron 2007;54:479-90.

33. Park CH, Chang WH, Lee M, Kwon GH, Kim L, Kim ST, et al. Predicting the performance of motor imagery in stroke patients: multivariate pattern analysis of functional MRI data. Neurorehabil Neural Repair 2015;29: 247-54.

34. Gale SD, Pearson CM. Neuroimaging predictors of stroke outcome: implications for neurorehabilitation. NeuroRehabilitation 2012;31:331-44.

35. Latchaw RE. Cerebral perfusion imaging in acute stroke. J Vasc Interv Radiol 2004;15(1 Pt 2):S29-46. 